\title{
Les défis du vécu sanitaire de jeunes bamakoisEs (Mali). Identifier les sources de vulnérabilité par la lunette des Capabilités.
}

\author{
Claudine Sauvain-Dugerdil et Mathias Lerch, \\ Institut d'études démographiques et du parcours de vie (I-DEMO), Université de Genève \\ Claudine.Sauvain@unige.ch
}

\section{Résumé}

Le cadre des Capabilités est appliqué à la santé des jeunes définie par trois indicateurs relevés lors d'une enquête par questionnaire auprès de 1819 BamakoisEs, de 12 à 29 ans. Nos résultats confirment que la jeunesse est une période en relative bonne santé, pas fonction du gradient socio-économique, mais avec un fort différentiel de genre. La richesse du contexte entraînerait des comportements masculins néfastes, alors que les jeunes femmes apparaissent sensibles à l'insalubrité de la zone de résidence. Les résultats soulignent aussi que la transition vers l'âge adulte est marquée par des seuils aux risques accrus: entrée à l'école et sur le marché du travail (surtout pour les hommes) et, pour les femmes, première maternité. Le recours au système moderne de soins ne constituerait pas une Capabilité, à savoir un facteur d'accroissement réel de la liberté de maintenir une bonne santé, mais serait plus conjoncturelle, et ne semble pas gommer les inégalités.

Mots-clés: santé, jeunes, Mali, inégalités socio-économiques, cadre de vie.

\section{Abstract}

\section{Challenges of the health experience of young people in Bamako (Mali). Identifying the sources of vulnerability through the Capability lens.}

We apply here the Capability framework to analyze the health situation measured by three indicators from a questionnaire survey on 1,819 young people aged 12 - 29 years in Bamako (Mali). Our results confirm that youth is a period of relatively good health, unaffected by socioeconomic status, but with important gender differentials. The wealth of the context appears to generate unhealthy behaviors among males, whereas young women appear to suffer more from an unhealthy living environment. Results show also that entering school and the labour market (especially for men), and female first pregnancy, are risky periods in the transition towards adulthood. Recourse to the modern health system does not appear to be a Capability, in the sense of a factor enhancing the freedom to conserve good health, but is rather occasional and does not seem to reduce health inequalities.

Keywords: health, youth, Mali, socio-economic disparities, living environement.

\section{Introduction}

La jeunesse est connue comme une phase de vie qui jouit globalement d'une bonne santé, mais c'est aussi une période caractérisée par un pic de surmortalité (Remund, 2012) et par l'adoption de comportements à risques qui peuvent avoir des conséquences à long terme (OMS 20II'). Ceci est d'autant plus vrai dans le contexte urbain africain. Jusqu'à récemment, l'urbanisation était considérée comme réduisant les risques de vecteurs infectieux, mais on 
reconnaît de plus en plus que, malgré une situation sanitaire globalement meilleure, les villes génèrent de grandes inégalités d'accès aux soins et des conditions de vie qui peuvent être néfastes. Dans les cités africaines, les problèmes de manque d'infrastructures d'assainissement et de pollution, et les traditions d'agriculture urbaine, entraînent en particulier une recrudescence du paludisme et autres vecteurs (Njama et al, 2003; Keiser et al 2004). C'est aussi en ville que les modes de vie se transforment le plus rapidement et que se développe ce qu'Amid Talaï et al (eds, 1995) avaient décrit comme la "Culture Jeunes", syncrétisme entre différentes influences qui conduit à une redéfinition des repères. Cette adhésion à une culture de pairs expliquerait que le gradient socio-économique de santé (Marmot, 2005) serait moins marqué parmi les adolescents (West, 1997 ; Holstein et al, 2009), thèse fondée sur des travaux relatifs aux populations occidentales que Griffith et al (2013) vérifie pour la montée de l'obésité en Afrique du Sud. La surmortalité des jeunes pourrait cependant refléter la situation de souspopulations vulnérables en matière de maladies de la pauvreté ou d'accidents (Remund, 2013).

Bien que l'importance des facteurs socioculturels soit de plus en plus reconnue (Lopez et al, eds 2006)ii, les développements d'une épidémiologie sociale (Heggenhougen et al., 2003) concerne essentiellement les sociétés occidentales et, comme le soulignent Oakes and Kaufmann (eds, 2006), souffrent de faiblesses méthodologiques, eu égard à la mesure des comportements sociaux et aux interprétations abusives de causalités. Si le rôle de la personne elle-même (agency) commence à être cité dans les modélisations (Glass et McAtee, 2006), la façon de le prendre en compte n'est pas résolue. D'autre part, les travaux relatifs à la santé des jeunes se concentrent sur les problèmes spécifiques liés à la santé sexuelle et de la reproduction, aux addictions, aux traumatismes, à la malnutrition et, plus récemment, à la santé mentale (OMS, 20ll). Malgré la reconnaissance des interdépendances entre ces différentes dimensions, il manque clairement des analyses considérant la santé des jeunes dans leur globalité, particulièrement en Afrique. Dans le contexte de précarité, d'incertitudes et de changements de valeurs des grandes villes africaines, la santé des jeunes pose des défis particuliers qui nécessitent de considérer sa multi-dimensionnalité et celle de ses déterminants, en particulier le rôle majeur des comportements et modes de vie.

Le présent travail a pour objectif d'analyser la situation globale de santé des jeunes, telle qu'ils/elles l'expriment à travers leurs déclarations relatives à leur vécu sanitaire. L'approche des Capabilités (Sen, 1999) fournit un cadre pour concevoir le vécu sanitaire comme un processus qui forge l'état de santé, dans lequel la personne joue un rôle central par ses capacités inégales à valoriser les ressources dont elle dispose. Par l'analyse dans cette optique des réponses fournies par de jeunes Bamakois-es à une enquête par questionnaire, nous ré-examinons ici les inégalités de santé à travers quatre postulats de base:

I. La définition large de la santé, comme état de bien-être, implique de prendre en compte sa nature multidimensionnelle, telle qu'elle est vécue par les individus dans leur contexte spécifique, ici à travers trois dimensions: la santé environnementale, l'histoire sanitaire individuelle et l'auto-évaluation.

2. Se centrer sur l'individu implique de considérer les facteurs de risques liés à ces différentes dimensions de la santé (WHO, 2009). En particulier, il s'agit de différencier les inégalités provenant des ressources du contexte, de celles dues aux caractéristiques de la personne et de son environnement proche et, dans le rôle de l'individu (agency), la part respective des dotations par rapport aux moyens, à savoir les facteurs d'inégalités qui peuvent être modifiés ou non.

3. Au-delà des facteurs biologiques, le fait d'être femme ou homme dans un contexte donné constitue un des axes 
centraux du différentiel des comportements de santé.

4. L'accès aux soins ne représente qu'un des déterminants de l'état de santé (Marmot et Wilkinson, eds 2006, OMS 2008) et son impact est particulièrement complexe lorsque le système sanitaire moderne reste déficient et entre en concurrence avec des traditions de recours à d'autres formes de soins.

\section{Données et cadre d'analyse}

Les choix faits dans cette étude reposent sur une dynamique de travaux de terrain ancrée dans le temps long de recherches de démographie anthropologique réalisées depuis trois décennies au Mali par l'Université de Genève avec ses partenaires maliens. Nous procédons ici à une analyse secondaire des données de l'enquête réalisée à Bamako en 2002 relative aux facteurs de vulnérabilité associés au passage à l'âge adulte en milieu urbainiii. L'enquête a interrogé 1819 jeunes des deux sexes, âgés de 12 à 29 ans dans trois zones peu privilégiées, par un échantillonnage systématique des ménages ${ }^{i v}$. Le questionnaire comportait six modules: module biographique relevant l'histoire de la scolarisation et des activités, les changements de domicile, les événements familiaux et les problèmes de santé; emploi du temps du samedi précédant l'enquête; entourage (composition de la concession, personnes à qui se confier, vie associative); santé et santé de la reproduction; caractéristiques des occupations; contexte socio-économique de la famille. La richesse de l'information (584 variables) permet donc d'examiner la santé dans la perspective large de l'histoire biographique et des modes de vie des jeunes.

Nous revenons ici sur cette enquête qui a déjà été l'objet d'une série de publications en particulier sur l'emploi du temps, la transition de la sexualité et la vulnérabilitév, pour examiner à la lumière de l'approche des Capabilités (AC) le volet de la biographie sanitaire qui avait été peu exploité dans les analyses précédentes ${ }^{\mathrm{vi}}$. L'accent mis par l'AC sur les opportunités est particulièrement riche pour traiter de la santé des jeunes telle qu'autodéclarée dans une enquête par questionnaire. Cette enquête n'avait pas été conçue dans l'optique de l'approche des Capabilités. C'est donc à postériori que nous construisons le cadre, c'est-à-dire que nous identifions les informations qui constituent les meilleurs indicateurs pour alimenter le modèle analytique (voir Figure I et les effectifs des sous-groupes dans le tableau 3).

La zone de résidence est considérée comme un indicateur des ressources du contexte. En effet, les trois zones ont été choisies en fonction d'une stratégie précise par rapport à leurs caractéristiques, particularités qui ont ensuite été confirmée lors d'un travail d'inventaire des ressources (Berthé, 2006). Niaréla et Sicoroni sont de taille similaire 27’256 habitants réparris sur 27 ha pour le premier et 30'000 sur 29 ha pour le second (dernier recensement avant l'enquête, soit 1998). Tous deux sont situés dans une région de la ville qui est antérieure aux récents développements urbanistiques de Bamako, mais ils forment un gradient centrepériphérie tant dans leur localisation géographique que dans leur urbanisme et leurs équipements. Niarela est un des plus anciens quartiers de Bamako. Les rues sont pavées et équipées de canaux d'évacuation des eaux, il est branché sur un réseau d'adduction d'eau et compte de nombreuses infrastructures, y compris une Mairie, une série de commerces formels, des hôtels, quatre bars, un night-club et deux terrains de sport, douze écoles primaire, trois du second cycle fondamental, deux lycées et une école professionnelle, un Centre de santé communautaire (CSCOM), un centre de Prévention materno-infantile (PMI), trois cliniques privées et cinq pharmacies. Sicoroni est un quartier spontané, sans urbanisme planifié qui s'étale au pied des collines, avec un grand marché mais peu d'infrastructures formelles. L'approvisionnement en eau se fait par quelques bornes fontaines et des puits. II compte huit 
écoles primaires, quatre du second cycle fondamental et un centre d'écoute communautaire; un CSCOM et deux pharmacies; un terrain de basket-ball et un de football, deux dojos clubs et un bar. BandiagaraCoura, secteur de Sicoroni situé sur les premiers contreforts de la colline, ne jouit d'aucune route carrossable, ni adduction d'eau, évacuation des eaux usées et réseau électrique et autres services. C'est un lieu d'accueil de migrants, en particulier Dogon. Ce gradient d'équipement en infrastructures est corroboré par les différences observées en matière de biens des ménages. En revanche, Sicoroni se distingue des deux autres zones d'éltude par salubrité moindre, notamment avec le plus d'eaux stagnantes.

Deux dimensions sont retenues en termes d'appartenance socio-culturelle. L'origine ethnique témoigne d'un ancrage dans des traditions culturelles distinctes, mais aussi des modes de vie qui différencient en particulier les éleveurs des agriculteurs, des traditions migratoires plus ou moins importantes et de liens, plus ou moins lointains et forts, avec une zone spécifique du pays dans laquelle le paludisme peut être endémique. L'enquête s'étant déroulée dans la capitale, située dans la zone Bambara, nous examinons dans quelle mesure une origine culturelle différente affecte la situation de santé en dépit du partage du même contexte de résidence. Nous distinguons ici cinq groupes, comparant les Bambaras (39\% de l'échantillon) aux ethnies d'éleveurs du Nord (17\%), aux autres groupes du Nord (13\%) et aux autres (26\%), c'est-à-dire les ethnies du Centre et Sud du pays, dans lesquelles on trouve les familles les plus riches et les plus scolarisées. La religiosité - déclarer une pratique régulière (57\% des jeunes interrogés) - constitue une autre facette de l'identité socioculturelle, particulièrement en une période de montée d'intégrismes et, pour les jeunes, de distanciation par rapport aux normes dominantes.

Trois variables fournissent des informations sur le contexte familial. La densité du réseau relationnel fournit un indice sur le rôle de conseil et de soutien des proches. Celui-ci a été construit à travers trois questions: être membre d'une association et/ou avoir des personnes à qui se confiervii et/ou des personnes avec lesquelles passer son samedi. Sur cette base, la moitié des individus ont un réseau relativement dense (58\%) $)^{\text {vii. }}$.

Nous retenons la taille de la concession qui, dans nos analyses précédentes, apparaissait plus pertinente que des mesures plus complexes de la structure familiale, telles que nucléarité, polygamie, nombre de jeunes. Les petits ménages reflèteraient une certaine modernité et les grands ménages à la fois l'attachement aux traditions de familles étendues et la capacité économique permettant d'accueillir la parenté (Locoh et Mouvagha-Sow, 2005); dans notre enquête, les ménages de taille intermédiaire apparaissent effectivement les moins aisés économiquement. La dimension économique est mesurée par un indicateur de confort du ménage, similaire à celui utilisé dans les EDS.

Les caractéristiques socio-démographiques habituelles sont retenues comme traits individuels: sexe, âge, niveau de formation, occupation. L'enquête ayant pour objectif d'analyser les étapes de la transition vers l'âge adulte, elle a ciblé trois périodes de vie qui, dans ce contexte de grand écart d'âge dans l'entrée dans la vie familiale, correspondent à des âges distincts selon le sexe: adolescent-e-s ( $12-15$ ans pour les filles et $15-19$ ans pour les garçons), jeunes (16-19 et 20-24), jeunes adultes, c'est-à-dire autour de la constitution de la famille (20-24 et 25-29 ans).

La scolarisation pour tous est loin d'être atteinte, même parmi les jeunes résidant à Bamako: dans la population étudiée ici, $38 \%$ de jeunes femmes et $33 \%$ de jeunes hommes n'ont jamais été à l'école. Nous incluons aussi le statut migratoire, variable essentielle dans le contexte d'émigration rurale vers les villes, mesurée par une biographie détaillée de la mobilité. La durée de résidence n'ayant pas d'influence sur la santé, nous distinguons ici 
simplement les jeunes qui ont toujours vécu à Bamako des migrants (35\% de l'échantillon).

Figure I. Application du cadre des Capabilités: les variables « proxy »

$\begin{array}{llll}\text { Ressources } & \text { Facteurs de } & \text { Capabilités : } & \text { Accomplissement : } \\ \text { du contexte } & \text { conversions } & \text { liberté de réaliser } & \text { réalisation }\end{array}$

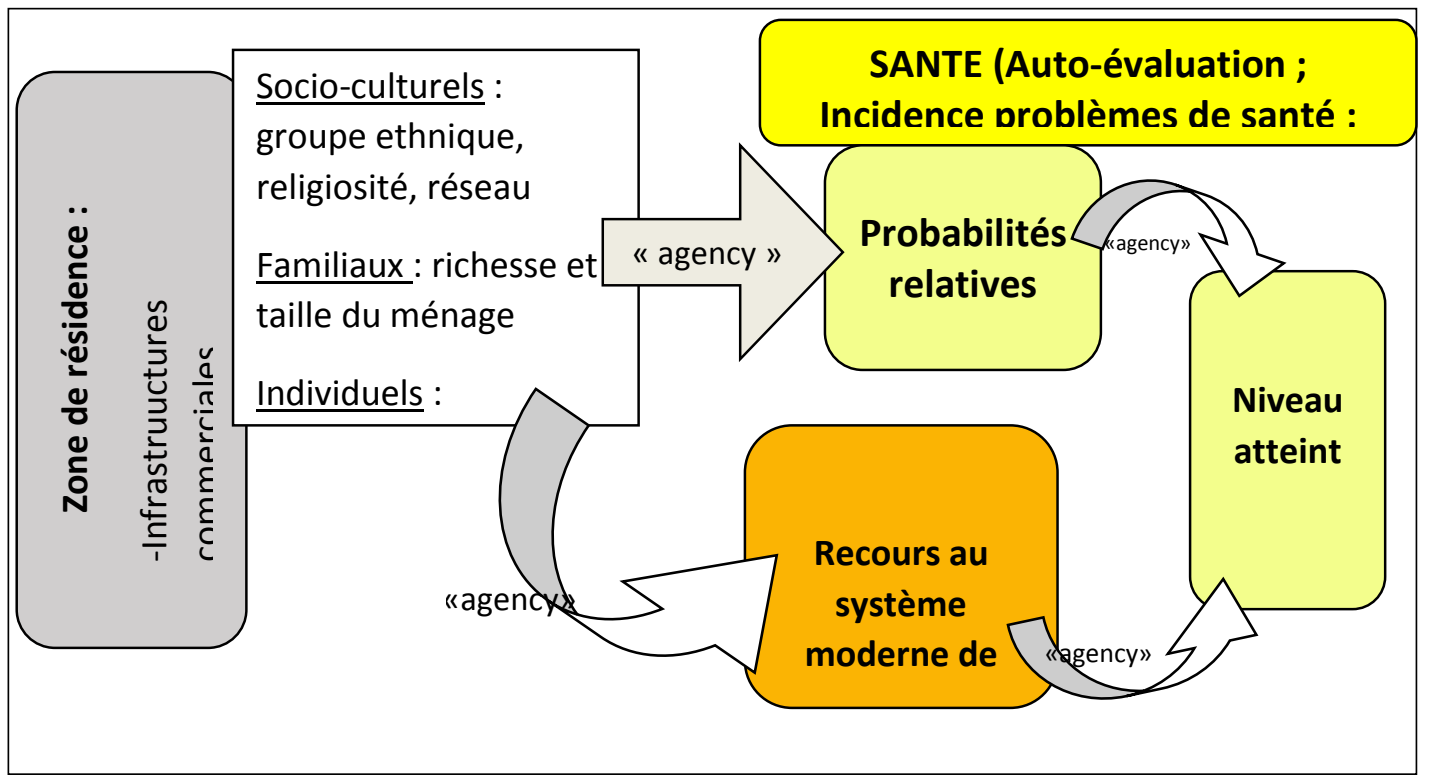

Adapté de Robeyns, 2005

Les disparités du vécu déclaré par les jeunes eu égard à leur histoire sanitaire, leurs itinéraires thérapeutiques et leur autoévaluation sont considérées comme des indicateurs de leur comportement sanitaire. L'hypothèse est que les épisodes de fièvre informent sur la santé environnementale et les comportements de prévention, alors que l'incidence des problèmes de santé en général reflèterait plus les inégalités résultant des disparités de ressources personnelles. Par sa subjectivité, l'auto-évaluation introduit les dimensions de préférence et d'ancrage socioculturel. Les niveaux atteints dans ses trois dimensions correspondent

aux accomplissements, alors que leur probabilité relative par rapport aux caractéristiques individuelles, telles qu'exprimées par les risques proportionnels dans l'analyse de régression, informe sur la possibilité d'atteindre une bonne santé, à savoir les Capabilités. Nous testons aussi la question de savoir si les jeunes recourent aux soins modernes avec l'intention de jouir d'une bonne santé, en d'autres termes est-ce que l'utilisation des services peut être considérée comme une Capabilité. Le rôle de l'individu (agency) est déduit par l'interprétation des divers types de facteurs d'inégalités d'opportunités et de réalisation.

\section{Résultats}

\section{Réalisation: la situation sanitaire des jeunes à la loupe de leurs déclarations}

Les problèmes de santé durant les cinq dernières années

Nous retenons ici les événements de santé déclarés $^{\mathrm{ix}}$ pour les cinq années précédant l'enquête (Tableaul). Une proportion importante des problèmes mentionnés se réfère aux crises aigues de paludisme. II est toutefois évident que même avec cette 
définition restrictive, nous n'avons pas là une mesure précise du taux de paludisme. D'une part, on n'a pas relevé les affections chroniques et, d'autre part, les déclarations rétrospectives ne fournissent évidemment pas d'informations sur les épisodes létaux. Finalement, il est bien connu que de nombreuses autres causes, telle des infections bactériennes graves, peuvent provoquer des épisodes fiévreux. Les travaux montrent en effet une grande variabilité de la proportion de paludisme avéré parmi les fièvres diagnostiquées $(4,6 \%$ à Luanda, $10 \%$ à Maputo et $20 \%$ à Ougadougou, Thwing et al, 2009; Macedo de Oliveira et al 20II). Par conséquent nous nous référerons ici à des épisodes fiévreux aigus sans conclure s'ils sont de type paludéen. $\mathrm{Vu}$ la formulation de la question, nous interprétons le nombre moyen annuel d'événements de santé comme un indicateur d'incidence. Certes, il est impossible de savoir si, au moment de l'événement de santé, les personnes étaient en bonne santé ou déjà malade, ou si certains problèmes seraient devenus chroniques. Toutefois, la durée n'étant que de 3 à 4 jours par année, nous estimons justifié de parler d'incidence.

Tableau I. Fréquence et durée moyenne des problèmes de santé et des épisodes aigus de fièvres. Cinq années précédant l'enquête (1998 - 2002).

\begin{tabular}{|c|c|c|c|c|c|c|c|c|c|}
\hline & \multicolumn{4}{|c|}{ FEMMES $(\mathrm{N}=942)$} & \multicolumn{4}{|c|}{ HOMMES $(\mathrm{N}=877)$} \\
\hline & & \multicolumn{2}{|c|}{ Santé } & \multicolumn{2}{|c|}{ Fièvre } & \multicolumn{2}{|c|}{ Santé } & \multicolumn{2}{|c|}{ Fièvre } \\
\hline & & $\begin{array}{c}\% \geq 1 \\
\text { problème }\end{array}$ & $\begin{array}{l}\text { Durée }^{x} \\
\text { (jours) }\end{array}$ & $\% \geq$ I épisode & $\begin{array}{l}\text { Durée } \\
\text { (jours) }\end{array}$ & $\begin{array}{c}\% \geq 1 \\
\text { problème }\end{array}$ & $\begin{array}{l}\text { Durée } \\
\text { (jours) }\end{array}$ & $\begin{array}{l}\% \geq 1 \\
\text { épisode }\end{array}$ & $\begin{array}{l}\text { Durée } \\
\text { (jours) }\end{array}$ \\
\hline Ado & $\begin{array}{l}\text { F I2-15 } \\
\text { H I5-19 ans }\end{array}$ & 55.2 & 43.1 & 31.2 & 49.8 & 50.5 & 41.1 & 24.4 & 38.9 \\
\hline Jeunes & $\begin{array}{l}\text { F I6- } 19 \\
\text { H 20-24 ans }\end{array}$ & 60.1 & 42.8 & 34.1 & 34.7 & 41.8 & 67.4 & 19.2 & 46.7 \\
\hline $\begin{array}{l}\text { Jeunes } \\
\text { adultes }\end{array}$ & $\begin{array}{l}\text { F 20-24 } \\
\text { H 25-29 }\end{array}$ & 60.2 & 39.2 & 26.9 & 33.2 & 31.4 & 64.3 & 11.2 & 55.6 \\
\hline & & $\mathrm{P}\left(\mathrm{Chi}^{2}\right)=.35$ & $P(F)=.86$ & $\begin{array}{l}\mathrm{P}\left(\mathrm{Chi}^{2}\right) \\
=.14\end{array}$ & $P(F)=.07$ & $\begin{array}{l}\mathrm{P}\left(\mathrm{Chi}^{2}\right) \\
=.000\end{array}$ & $\begin{array}{l}\mathrm{P}(\mathrm{F}) \\
=.07\end{array}$ & $\begin{array}{l}\mathrm{P}\left(\mathrm{Chi}^{2}\right) \\
=.000\end{array}$ & $P(F)=.71$ \\
\hline
\end{tabular}

La thèse de la jeunesse comme une période de vie en bonne santé est confirmée ici par la faible incidence de problèmes. La moitié des jeunes $(49,7 \%)$ n'a déclaré aucun problème durant ces cinq années, $42,3 \%$ un problème et seuls $8 \%$ plus d'un problème; respectivement $67,5 \%$ n'a déclaré aucun épisode fiévreux, $31,9 \%$ un épisode et $0.5 \%$ plus d'un épisode. D'autre part, on n'observe pas de cumul de problèmes; au contraire, toutes choses égales par ailleurs, le fait d'avoir déjà connu un problème de santé auparavant diminue considérablement le risque pendant les cinq dernières années.

$\mathrm{Vu}$ cette faible incidence, nous retenons comme indicateur le fait d'avoir connu, ou non, au moins un problème, ou un épisode, durant les cinq années avant l'enquête. Les femmes déclarent plus de problèmes mais, à âge équivalent, leur durée est nettement moindre. Les hommes auraient donc tendance à déclarer moins de problèmes, mais des problèmes plus 
graves. On observe aussi, pour les hommes, une diminution de l'incidence avec l'âge, l'effet étant plus complexe pour les femmes. Les plus âgées ont effectivement une fréquence un peu moindre d'épisodes fiévreux, mais ce sont au contraire les plus jeunes qui ont un peu moins de problèmes de santé. Contrairement aux hommes, pour les femmes la durée ne s'accroît pas avec l'âge; on peut penser que les charges domestiques croissantes qu'elles doivent assumer les empêchent de restreindre leurs activités même en cas de maladie.

\section{Santé générale auto-évaluée.}

Cet indicateur d'auto-perception ${ }^{\times i}$ est à la fois apprécié comme la meilleure mesure synthétique, reconnu comme bon prédicteur de mortalité, et critiqué car difficile à interpréter puisqu'il reflète un point de vue individuel sur l'état tant physique, qu'émotionnel et psychologique, influencé par les circonstances du moment (Idler and Benyamini 1997, Uttley and Crawford 2005). Sur la base d'une analyse des enquêtes mondiales de santé de 69 pays,
Subramanian et al. (2010) concluent sur la pertinence de cet indicateur, notamment en réfutant la thèse de biais dus aux sousdéclarations des groupes désavantagés.

Le bon état de santé des jeunes interrogés est corroboré par une bonne auto-évaluation: un peu plus de la moitié des jeunes s'estiment en très bonne santé, alors que seuls 31 jeunes se sont déclarés en mauvaise santé (1.7\%), catégorie que nous avons donc regroupé avec la modalité «moyenne» parmi les «médiocre». L'auto-évaluation est significativement associée à l'incidence de problèmes de santé: près des trois-quarts des jeunes n'ayant déclaré aucun problème de santé s'estiment en très bonne santé, cependant près de la moitié de ceux qui ont vécu deux problèmes s'estiment aussi en très bonne santé (Tableau 2). L'association est moindre pour les épisodes fiévreux qui n'auraient donc pas eu de conséquences suffisamment graves pour influencer l'autoévaluation au moment de l'enquête.

Tableau 2. Histoire sanitaire et auto-évaluation

\begin{tabular}{|c|c|c|c|c|c|c|c|c|}
\hline \multirow[b]{3}{*}{ Auto-évaluation } & \multicolumn{7}{|c|}{ Nombre de problèmes déclarés } & \\
\hline & \multicolumn{4}{|c|}{ Problèmes de santé } & \multicolumn{3}{|c|}{ Episodes aigus de fièvre } & \\
\hline & $\mathbf{0}$ & I & 2 & $3+$ & $\mathbf{0}$ & I & $2+$ & Total \\
\hline Très bonne & $73 \%$ & $48 \%$ & $49 \%$ & $41 \%$ & $57 \%$ & $51 \%$ & $56 \%$ & $55 \%$ \\
\hline Bonne & $20 \%$ & $28 \%$ & $27 \%$ & $36 \%$ & $25 \%$ & $27 \%$ & $11 \%$ & $26 \%$ \\
\hline $\begin{array}{l}\text { Moyenne- } \\
\text { mauvaise }\end{array}$ & $7 \%$ & $25 \%$ & $24 \%$ & $24 \%$ & $18 \%$ & $23 \%$ & $33 \%$ & $19 \%$ \\
\hline Total & $100 \%$ & $100 \%$ & $100 \%$ & $100 \%$ & $100 \%$ & $100 \%$ & $100 \%$ & $100 \%$ \\
\hline \multirow[t]{2}{*}{$\mathrm{N}$} & 536 & 945 & 279 & 42 & 1219 & 574 & 9 & 1802 \\
\hline & \multicolumn{4}{|c|}{$\mathrm{Chi}^{2} \mathrm{P}=0.000$} & \multicolumn{3}{|c|}{$\mathrm{Chi}^{2} \mathrm{P}=0.07$} & \\
\hline
\end{tabular}

\section{Les Capabilités:}

\section{Facteurs d'inégalité: les probabilités relatives de jouir d'une bonne santé}

Par des analyses de régression logistique, nous examinons les probabilités relative de jouir d'une bonne santé selon les des caractéristiques retenues (Tableau 3). Nos travaux antérieurs ayant confirmé que, même parmi les jeunes urbains, les différences de genre ne sont pas gommées et qu'elles portent non seulement sur les situations de vie, mais aussi sur l'importance relative de différentes caractéristiques, telles la scolarisation et le niveau socio-économique (Sauvain-Dugerdil et al, 2005 \& 2008), nous procédons à des analyses séparées pour les jeunes hommes et les jeunes femmes..

\section{a) Les ressources du contexte}


Les résultats soulignent que le lieu de résidence est un déterminant significatif de la situation sanitaire des jeunes, ceci même lorsque l'on contrôle pour les inégalités socioculturelles, familiales et individuelles, mais qu'il s'agit d'un indicateur multi-dimensionnel. Les résultats sont cohérents avec le degré de salubrité. Ainsi, pour les femmes, les épisodes fiévreux apparaissent significativement moins fréquents à Bandiagara Coura et à Niarela qu'à Sicoroni. On observe aussi un léger avantage pour les hommes résidant à Bandiagara Coura, l'effet perdant toutefois sa significativité statistique après le contrôle pour l'occupation.

En revanche, pour cette population de jeunes, on n'observe pas de relation simple et positive avec la richesse relative des trois zones. Ainsi, ce sont les jeunes hommes de Niarela, le quartier le plus favorisé, qui ont la moins bonne santé: l'incidence des problèmes y est $50 \%$ plus élevée que dans les deux autres zones. On peut penser que dans ce contexte d'offre plus abondante en commerces et bars de toutes sortes, les jeunes adopteraient un mode de vie moins favorable à leur santé. Ceci corrobore nos résultats antérieurs relatifs à l'adoption plus marquée chez les jeunes hommes de ce quartier de comportements à risques en matière de sexualité, et de consommation de tabac et d'alcool (Berthé et al, 2005).

\section{b) Facteurs de conversion socio- culturels et familiaux}

Pour les femmes, l'appartenance ethnique et la pratique religieuse ne jouent guère de rôle. Parmi, les jeunes hommes, les non-éleveurs du Nord se distinguent par une meilleure situation sanitaire, avec les coefficients les plus bas en termes d'incidence de problèmes de santé et d'épisodes fiévreux aigus, et une autoévaluation légèrement meilleure. Les Dogon, fortement représentés parmi les non-éleveurs du Nord, auraient donc un net avantage sanitaire provenant des caractéristiques de cette population. Rappelons que c'est le groupe qui au niveau national souffre le moins de déficit pondéral, alors que c'est parmi les éleveurs Peuhls que la prévalence est la plus élevée (EDS, 2006). Les jeunes les plus religieux tendent à avoir une meilleure santé, mais seul l'effet sur l'incidence des problèmes de santé des hommes est statistiquement significatif.

Le fait d'avoir un entourage plus dense constituerait un appoint pour la santé. II améliore légèrement la perception que les femmes ont de leur santé, toutefois l'effet perd sa significativité en introduisant le contrôle pour les caractéristiques individuelles. Pour les hommes, on observe une corrélation positive, mais statistiquement non significative entre la santé et le niveau de vie du ménage. En revanche, pour les femmes, le fait de vivre dans les familles les plus aisées apparaît être un facteur de moins bonne santé. Bien que non significative statistiquement, la relation va dans le même sens en matière d'auto-estimation de leur état de santé et d'épisodes fiévreux. La taille du ménage n'a pas d'effet sur la santé des jeunes femmes et, pour les hommes, perd sa significativité statistique en contrôlant pour la scolarisation, les grands ménages ayant en leur sein plus de non-scolarisés. 
Tableau 3. Facteurs d'inégalité de santé.

Régression logistique sur trois dimensions de la santé auto-déclarée

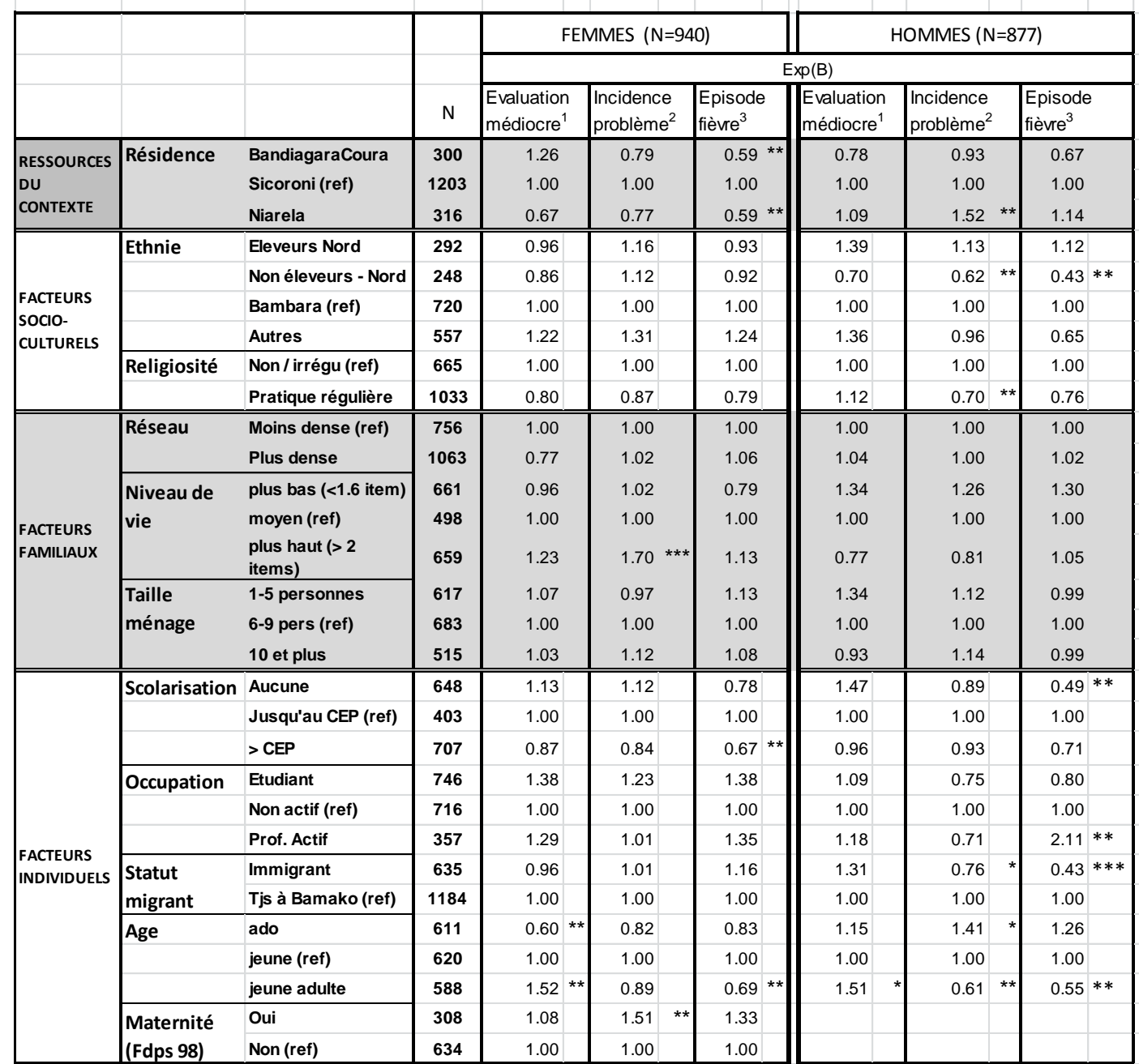

Régression logistique, valeur de $\operatorname{Exp}(\mathrm{B}):{ }^{* * * \mathrm{P}<.01},{ }^{* * \mathrm{P}<}<.05,{ }^{*} \mathrm{P}<.10$

${ }^{1}$ Jeunes ayant déclaré leur santé "moyenne" ou "mauvaise" (189 F / 159 H)

${ }^{2}$ Jeunes ayant déclaré avoir eu au moins un problème de santé au cours des 5 années précédant l'enquête $(549 \mathrm{~F} / 364 \mathrm{H})$

${ }^{3}$ Jeunes ayant déclaré avoir eu au moins un épisode aigü de fièvre au cours des 5 années précédant l'enquête ( $288 \mathrm{~F}$ et $162 \mathrm{H}$ )

\section{a) Facteurs individuels}

Les ressources fournies par la scolarisation et l'occupation

La scolarisation et les activités professionnelles ont des effets complexes d'acquisition de compétences et d'ouverture en dehors du cadre familial. C'est bien le cas dans la présente analyse qui ne montre pas de parallèle simple entre la santé et le gradient de scolarisation. Globalement, pour les deux sexes, on observe un effet en $U$ corroborant la thèse des risques liés à l'ouverture sur l'extérieur: les jeunes n'ayant suivi que quelques années d'école ont une moins bonne santé que ceux qui ne l'ont jamais fréquentée ou, au contraire, ont fait de plus longues études.

Le fait d'avoir une occupation a un effet propre important même en contrôlant pour les autres caractéristiques, notamment celui du niveau de scolarisation. L'incidence des épisodes fiévreux aigus est accrue par une occupation hors du foyer, et ceci encore plus pour les hommes. On peut donc penser que 
l'occupation augmente le contact avec les vecteurs des pathologies fiévreuses.

Les caractéristiques liées à l'histoire de vie

Pour les femmes, le statut migratoire n'a pas d'effet. Alors que parmi les hommes, le fait de ne pas avoir toujours vécu à Bamako réduit considérablement l'incidence de problèmes de santé, particulièrement celle des épisodes fiévreux; cet avantage peut résulter d'une résistance acquise à certaines pathologies ou d'un effet sélectif. Notons aussi que chez les garçons, l'effet des longues études a perdu de son intensité en contrôlant pour le statut de migration; le statut migratoire (et l'occupation) diminuent aussi légèrement l'effet du contexte et de l'appartenance ethnique.

Pour les deux sexes, la santé subjective se dégrade considérablement avec la progression vers l'âge adulte. Ce gradient ne se retrouve pas en termes de santé. Chez les hommes, on observe même une tendance inverse de diminution importante des problèmes de santé et des épisodes fiévreux avec l'âge. On peut donc faire l'hypothèse que les plus jeunes seraient plus vulnérables et/ou adopteraient des comportements moins sains que leurs aînés; ces derniers auraient certes une vision plus critique de leur santé, mais prendraient mieux soins d'eux-mêmes et/ou auraient acquis une certaine résistance.

Pour les femmes, l'avancement en âge se confond avec les problèmes de santé associés à la maternité. Si le vécu d'une grossesse ne modifie pas leur jugement sur leur état de santé, en revanche la maternité accroît considérablement l'incidence de problèmes de santé et, dans une moindre mesure, celle des épisodes fiévreux.

\section{Recours au système moderne de soins}

Le recours au système moderne de soins ${ }^{\mathrm{xii}}$ n'est pas systématique: à peine plus de la moitié des jeunes $(55,7 \%)$ ont recouru au moins une fois lors d'un problème au cours des cinq années précédant l'enquête, soit $47 \%$ des hommes et $61.5 \%$ des femmes. Ces chiffres sont légèrement inférieurs à ceux rapportés dans l'EDS 2006 (59,2\% des malades résidant à Bamako). Dans $14,1 \%$ des cas, le premier recours fut un médecin traditionnel.

Pour les deux sexes, les caractéristiques socio-culturelles, la taille du ménage et l'âge n'ont pas d'effet sur le recours aux soins (Tableau 4, deux premières colonnes). Les immigrants utilisent un peu moins le système moderne, particulièrement chez les hommes, sans toutefois atteindre la significativité statistique.

Parmi les caractéristiques considérées, seules la scolarisation et l'occupation ont un effet significatif pour les deux sexes. La fréquentation scolaire conduirait à un recours accru au système de soins, confirmant d'autres travaux sur l'effet d'une meilleure connaissance des causes et symptômes sur la propension des citadins à faire soigner leurs enfants fiévreux à I'hôpital (Njama et al, 2003; Rossier et al, 20l I), ou un recours plus précoce au système de soins qui diminue le risque d'aggravation d'une affection légère (Safeukui-Noubissi et al, 2004). L'utilisation est en outre accrue par l'exercice d'une activité professionnelle, qui comme la fréquentation de l'école est un facteur important de contact avec le monde et les idées de l'extérieur. Le contrôle pour l'effet du statut d'occupation accroît aussi l'impact négatif de l'absence de scolarisation, ainsi l'activité professionnelle aurait donc en matière de recours aux soins, un effet compensatoire au manque de scolarisation.

Pour les hommes, avoir un réseau relationnel plus dense est un facteur d'utilisation accrue: ainsi, les proches peuvent donc jouer un rôle, mais de façon sélective. Une analyse plus détaillée avait montré que le recours était plus fréquent lorsque le père est une figure centrale des personnes ressources en matière de santé (Berthé et al, 2008). Le contexte de résidence et le niveau de vie du ménage n'influencent pas le recours des hommes aux services modernes, mais ont des effets de prime abord paradoxaux 
pour les femmes. Ce sont en effet celles qui résident dans la zone la moins privilégiée Bandiagara Coura - et qui proviennent des familles les moins aisées qui utilisent le plus les services de santé lorsqu'elles ont un problème. Ainsi, toutes choses égales par ailleurs, la richesse et la proximité des services n'apparaît pas comme un facteur d'utilisation. Pour les femmes, c'est au contraire une situation de privation - de services de proximité et de ressources - qui les amènerait à se déplacer. Cet effet de pauvreté relative s'accroît lorsque l'on introduit le contrôle pour la scolarisation et le statut d'occupation, c'est-à-dire dans le cas de Bandiagara Coura, l'expérience de mobilité hors de la zone de résidence.

Globalement, pour les deux sexes, un recours aux soins lors d'un problème antérieur est associé à une utilisation ultérieure moindre, sauf pour les femmes de Bandiagara Coura dont l'utilisation est encore renforcée (résultats non figurés ici). Pour les femmes, un recours antérieur accroît en outre le risque de problèmes subséquents (pas d'effet significatif pour les hommes): un premier contact avec le système moderne de soins pourrait donc entraîner une prise de conscience accrue de sa santé qui s'exprime dans l'accroissement des problèmes déclarés et un recours à l'automédication pour des problèmes de moindre importance. Cette hypothèse est corroborée par l'impact du premier contact avec les services sur les facteurs d'inégalités de santé ultérieurs (Tableau 4, quatre dernières colonnes). L'utilisation des services gomme l'effet du niveau de vie, mais amplifie celui de l'absence de scolarisation. Cette dernière a une influence opposée selon le sexe. Pour les filles, un contact antérieur avec les services accroît le désavantage sanitaire des moins scolarisées, mais avantage les filles exerçant une activité professionnelle. Parmi les garçons, les non scolarisés et les jeunes ayant un réseau relationnel dense ont une moindre incidence de problèmes ultérieurs lorsqu'ils ont eu un contact avec les systèmes de soins, ils bénéficieraient donc plus des compétences fournies dans les centres de santé.

\section{Tableau 4. Recours au système de soins.}

Facteurs d'inégalités du recours au système de soins et effets d'un recours antérieur sur l'incidence des problèmes de santé.

\begin{tabular}{|c|c|c|c|c|c|c|c|c|}
\hline & & & \multicolumn{2}{|c|}{$\begin{array}{l}\text { Recours au sytème de } \\
\text { soins durant les cinq } \\
\text { dernières années }^{1}\end{array}$} & \multicolumn{4}{|c|}{$\begin{array}{l}\text { Problèmes de santé durant les cinq dernières } \\
\text { années, avec/sans recours au système de soins } \\
\text { pour un problème antérieur }{ }^{2}\end{array}$} \\
\hline & & & \multirow{2}{*}{$\begin{array}{l}\text { FEMMES } \\
(\mathrm{N}=551)\end{array}$} & \multirow{2}{*}{$\begin{array}{l}\text { HOMMES } \\
(\mathrm{N}=364)\end{array}$} & \multicolumn{2}{|c|}{ FEMMES ( $\mathrm{N}=195)$} & \multicolumn{2}{|c|}{ HOMMES (314) } \\
\hline & & & & & Avec $(\mathrm{N}=108)$ & Sans $(N=87)$ & Avec $(\mathrm{N}=159)$ & Sans $(\mathrm{N}=155)$ \\
\hline \multirow{3}{*}{$\begin{array}{l}\text { RESSOURCES } \\
\text { DU CONTEXTE }\end{array}$} & Résidence & Bandiagara Coura & $1.74^{* *}$ & 0.94 & 2.07 & 1.78 & 0.95 & 1.46 \\
\hline & & Sicoroni (ref) & 1 & 1 & 1 & 1 & 1 & 1 \\
\hline & & Niarela & 1.23 & 0.91 & 0.80 & 1.29 & 0.99 & 1.17 \\
\hline \multirow{6}{*}{$\begin{array}{l}\text { FACTEURS } \\
\text { SOCIO- } \\
\text { CULTURELS }\end{array}$} & Ethnie & Eleveurs Nord & 1.13 & 1.15 & $3.98^{* \star}$ & 5.83 * & 1.18 & 0.54 \\
\hline & & Non éleveurs-Nord & 0.95 & 0.74 & 3.27 & 2.54 & 0.75 & 0.50 \\
\hline & & Bambara (ref) & 1 & 1 & 1 & 1 & 1 & 1 \\
\hline & & Autres & 1.37 & 1.06 & 1.30 & 2.09 & 2.39 * & 0.37 * \\
\hline & Religiosité & Non / irrégu (ref) & 1 & 1 & 1 & 1 & 1 & 1 \\
\hline & & Pratique régulière & 1.00 & 1.12 & 1.15 & 0.79 & 0.83 & 0.51 \\
\hline \multirow{5}{*}{$\begin{array}{l}\text { FACTEURS } \\
\text { FAMILIAUX }\end{array}$} & Réseau & Moins dense (ref) & 1 & 1 & 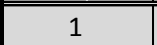 & 1 & 1 & 1 \\
\hline & & Plus dense & 0.87 & 1.70 ** & 1.05 & 0.84 & $0.35 * *$ & 0.63 \\
\hline & Niveau de & plus bas & $1.53 *$ & 0.83 & 0.53 & 1.90 & 1.78 & 0.35 * \\
\hline & & moyen (ref) & 1 & 1 & 1 & 1 & 1 & 1 \\
\hline & & plus haut & 1.05 & 0.88 & 1.61 & 9.38 ** & 1.75 & 0.90 \\
\hline
\end{tabular}




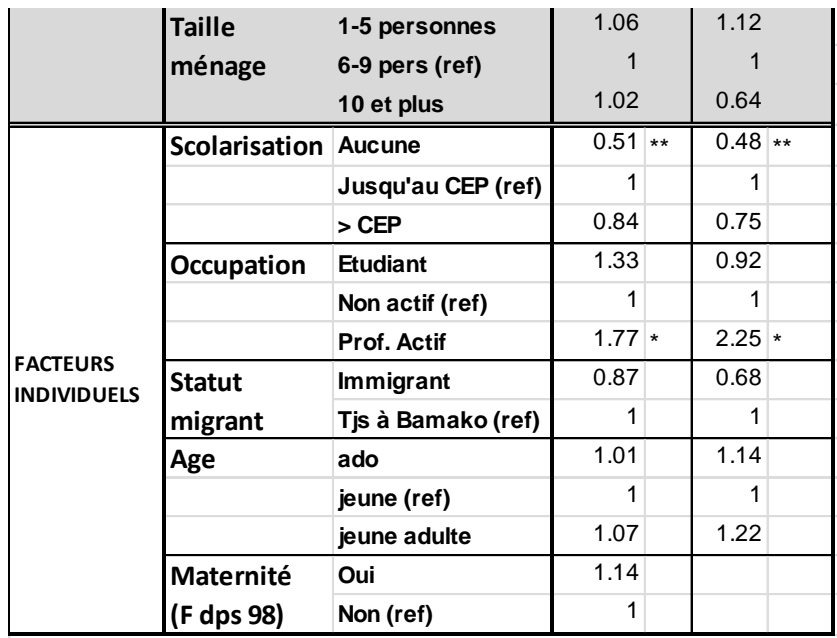

\begin{tabular}{||r|r|r|r|}
0.59 & 1.21 & 1.04 & 1.55 \\
1 & 1 & 1 & 1 \\
0.86 & 0.92 & 1.15 & 1.09 \\
\hline \hline $4.93 *$ & 0.50 & $0.12 *$ & 1.15 \\
1 & 1 & 1 & 1 \\
\hline 1.41 & 1.21 & 1.12 & 0.66 \\
\hline 0.65 & 1.33 & 1.08 & 1.40 \\
\hline 1 & 1 & 1 & 1 \\
\hline $0.09 * *$ & 2.18 & 5.59 & 0.52 \\
\hline 0.61 & 2.46 & 0.74 & 0.47 \\
\hline 1 & 1 & 1 & 1 \\
\hline 1.30 & 0.64 & 0.85 & 1.31 \\
\hline 1 & 1 & 1 & 1 \\
\hline 0.91 & 0.45 & 0.44 & 1.38 \\
\hline $5.17 * \star \star$ & 1.20 & & \\
\hline 1 & 1 & & \\
\hline
\end{tabular}

Régression logistique, valeur de $\operatorname{Exp}(\mathrm{B}):{ }^{* *} \mathrm{P}<.01,{ }^{*} \mathrm{P}<.05,{ }^{*} \mathrm{P}<.10$

${ }^{1}$ Jeunes ayant eu au moins un problème de santé durant les 5 dernières années

2 Jeunes ayant eu au moins un problème antérieur de santé

\section{Discussion}

En appliquant le cadre des Capabilités à l'analyse du vécu sanitaire tel que relevé dans une enquête par questionnaire, l'objectif est d'identifier les facteurs de vulnérabilité dans une population de jeunes bamakoisEs. Par le type de données et le cadre conceptuel choisi, l'accent est mis sur le processus - la liberté de jouir d'une bonne santé - tel que vécu par la personne et non pas le résultat dans une optique médicale. Une analyse fine des facteurs de risques a été réalisée en distinguant la variabilité due au contexte de celle due à diverses caractéristiques socio-culturelles, familiales et personnelles relatives à quatre dimensions du vécu sanitaire - l'incidence globale des problèmes de santé, les épisodes de fièvres, l'auto-évaluation et le recours au système moderne de soins.

II convient cependant de garder à l'esprit les limites du présent travail. L'enquête n'ayant pas été conçues dans l'optique des Capabilités, c'est à posteriori que l'on a identifié les indicateurs les plus pertinents pour la modélisation. Le type de données récoltées, mais aussi la taille de l'échantillon, ne permettent pas de procéder à des analyses statistiques plus sophistiquées (multi-niveau, tests d'interactions). Notons aussi qu'il n'est pas possible de généraliser les résultats puisque la population enquêtée n'est représentative que des zones de Bamako considérées ici et que l'absence de travaux similaires, empêche de les situer par rapport à d'autres populations. Les résultats restent donc de nature exploratoire et sont à considérer comme des hypothèses à tester sur d'autres terrains.

Nos résultats confirment que, si les jeunes jouissent globalement d'une bonne santé, en revanche, ils peuvent adopter des comportements néfastes susceptibles d'entraîner, «à un stade ultérieur de la vie, une

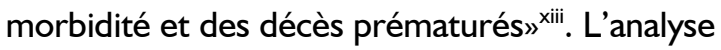
met en évidence les transformations du vécu au long de la transition vers l'âge adulte, la vulnérabilité aux conditions du contexte et les fortes différences de genre à cet égard, le maintien même en milieu urbain des différences dues aux origines culturelles, les effets complexes des ressources personnelles et de l'accès au système moderne de soins.

\section{Entrée dans l'âge adulte: une transition} sanitaire?

La préoccupation pour la santé semble se construire durant la transition vers l'âge adulte. Pour les plus jeunes interrogés dans cette enquête (filles de 12-15 ans et garçons de 1519), la santé ne semble pas constituer une 
préoccupation centrale. Leur auto-évaluation est en effet meilleure que chez les plus âgés, bien qu'ils déclarent plus de problèmes de santé. Les plus âgés sont plus critiques sur leur santé, alors que leur morbidité déclarée est moindre. Au-delà de l'avancement en âge en tant que tel, la transition vers l'âge adulte est marquée par des seuils: pour les femmes, le vécu d'une maternité entraîne une fréquence accrue de problèmes de santé, et, pour les deux sexes (encore plus pour les hommes), l'entrée dans le marché du travail apparaît associée à une incidence plus forte d'épisodes fiévreux aigus.

Les sources d'inégalités de santé des femmes et des hommes

De fortes différences de santé confirment que le milieu urbain ne gomme pas les inégalités de genre. Chez les jeunes femmes, la morbidité apparait plus forte, mais plus homogène; la santé des jeunes hommes semble en effet plus influencée par les caractéristiques considérées ici ${ }^{\text {iv }}$. On constate aussi que les facteurs d'inégalités ne sont pas les mêmes, ou n'agissent pas dans le même sens. Seuls, l'avancement en âge et l'effet en $U$ de la scolarisation affectent les deux sexes de façon similaire. Le lieu de résidence a des effets différents et ce n'est que pour les hommes que les modes de vie acquis et/ou les effets sélectifs - tels qu'exprimés par l'origine socio-culturelle et le statut migratoire jouent un rôle significatif. En revanche, ce n'est que pour les femmes que compte le niveau de vie du ménage

La salubrité du contexte, un facteur important de la prévalence des fièvres aiguës

Même en contrôlant pour les différences socio-culturelles, familiales et individuelles, le lieu de résidence reste un déterminant important de la santé. Pour les jeunes femmes, l'environnement joue un rôle majeur, avec près de deux fois plus d'épisodes fiévreux aigus dans la zone la moins salubre. Ceci confirme la plus grande vulnérabilité féminine à l'environnement, due au moins en partie à leur contact fréquents avec les eaux, à travers leurs tâches domestiques. Comme nous l'avons déjà relevé, l'exercice d'une activité professionnelle est aussi associé à un eplus forte incidence des fièvres, vraisemblablement par une exposition accrue aux vecteurs; cet effet est plus marqué pour les jeunes hommes ce qui pourrait résulter d'activités plus à risque à cet égard ${ }^{\mathrm{x} v}$.

En milieu urbain, les ressources économiques ne constitueraient pas un obstacle à la santé, mais auraient une influence indirecte à travers des modes de vie distincts

On n'observe pas de relation entre le gradient économique des zones et la situation de santé des jeunes résidants. Pour les jeunes hommes, au contraire, c'est le fait de vivre dans la zone la plus riche et la plus salubre qui est associé à une incidence considérablement plus forte de problèmes de santé. Les jeunes hommes de ce quartier, doté en commerces et bars de toutes sortes, adopteraient donc des comportements plus à risques.

Pour les jeunes femmes, le fait de vivre dans un quartier plus aisé n'implique pas de modes de vie plus à risques, ce qui est consistant avec un contrôle familial plus marqué des filles et leur fréquentation moindre des lieux publics ${ }^{x v i}$. Cependant, globalement, les jeunes femmes les moins favorisées - des quartiers et de familles les plus pauvres - auraient une meilleure santé. On peut donc penser que, dans cette population de jeunes qui, rappelons-le, ne comprend pas les jeunes marginaux sans logis stables, le manque de moyens économiques ne serait pas un obstacle à la jouissance d'une bonne santé et que les moins aisées adoptent des comportements plus favorables.

Pour les jeunes hommes, importance de l'ancrage socio-culturel

Pour les jeunes hommes, la résidence dans la capitale n'a pas gommé les différences liées à l'origine culturelle, toutefois l'effet est complexe. En particulier, on n'observe pas de gradient clair eu égard au degré d'endémie du paludisme dans les zones d'origine ethnique 
(Larochelle, 2004). D'autre part, la meilleure santé des jeunes n'ayant pas toujours vécu à Bamako est cohérente avec les thèses d'effet sélectif de la migration: les migrants étant ceux qui jouissent d'un meilleur potentiel. Cet effet sélectif ne concerne cependant que les hommes.

\section{Scolarisation: risques et compétences}

Nos résultats confirment l'effet ambigu que peut avoir l'accès à l'école. Pour les deux sexes, la scolarisation a un effet en $U$ sur l'incidence des épisodes fiévreux, à savoir une moins bonne santé parmi ceux qui ont fréquenté l'école juste quelques années (en comparaison avec ceux qui ne sont jamais allés à l'école et ceux qui y sont resté au-delà du primaire). Cet effet est cohérent avec la thèse des risques associés aux contacts avec l'extérieur et à l'adoption de comportements pouvant être néfastes à la santé. Parmi les plus scolarisés, particulièrement pour les filles, ces risques seraient réduits par l'acquisition de nouvelles compétences liées à une scolarisation plus longue.

L'accès aux soins diminue le risque sanitaire, mais ne gomme pas les inégalités de santé

L'absence d'effets de l'âge sur le recours au système de soins montre qu'il n'y aurait pas de discrimination des plus jeunes en matière d'accueil comme c'est le cas pour les services de santé de la reproduction. Surtout, nos résultats témoignent qu'il n'y a pas de relation simple et directe entre la situation sanitaire et le recours aux services. Ce dernier n'est pas forcément l'expression d'un mode de vie propice à la santé ou inversement la conséquence d'une moins bonne santé. On observe certes une cohérence entre l'accès accru et une meilleure santé pour des sousgroupes, tels que les femmes les plus pauvres et du quartier le moins aisé, mais aussi une meilleure santé parmi les plus scolarisées. Les jeunes non scolarisés recourent moins au système de santé, mais ils déclarent moins d'épisodes fiévreux (surtout les jeunes hommes). En revanche, pour les deux sexes, l'activité professionnelle diminue la qualité de la santé et accroît le recours aux services. Et si, pour les jeunes hommes, un réseau relationnel plus dense stimule le recours aux services, il n'aurait pas d'effet bénéfique sur leurs comportements sanitaires. Ces incohérences semblent indiquer que l'utilisation des services de santé ne serait pas l'expression d'une préoccupation particulière pour sa santé, mais serait conjoncturelle, liée à d'autres facteurs: elle apparaît stimulée par la mobilité accrue des jeunes actifs professionnellement et, pour les jeunes hommes, l'incitation donnée par un réseau plus dense et elle serait freinée par un faible niveau de connaissances.

En second lieu, rien ne justifie une interprétation causale de l'association négative entre une utilisation du système moderne de santé et le risque d'avoir un problème subséquent de santé ou de moindre recours lors d'un nouveau problème. D'une part, ces résultats ne concernent évidement que le petit groupe de jeunes qui se distingue par une santé moins bonne et qui a donc un vécu sanitaire particulier. D'autre part, l'effet négatif de ce premier contact sur la santé ultérieure n'est pas homogène. Parmi les femmes ayant eu une expérience antérieure avec les services, ce sont les non scolarisées ou celles qui ont connu une maternité, qui ont une probabilité accrue d'avoir un nouveau problème de santé. En revanche, ce contact semble bénéficier à celles des ménages plus aisés ou qui ont acquis des compétences par une activité professionnelle. Ainsi, elles adopteraient des comportements moins sains (risque accru de problèmes de santé et moindre utilisation des services), mais elles bénéficieraient plus du contact avec les services lorsqu'elles y recourent. On peut donc émettre l'hypothèse selon laquelle, les services semblent bénéficier aux jeunes femmes qui ont déjà certaines ressources et, au contraire, accroître le handicap des moins bien loties eu égard à leur situation de santé et à leurs compétences.

Pour les hommes, un contact antérieur ne modifie globalement pas le risque sanitaire 
subséquent, mais des inégalités subsistent qui diffèrent de celles des femmes. C'est aux non scolarisés et aux jeunes bénéficiant d'un réseau relationnel dense que ce contact donne un avantage sanitaire et aux actifs au contraire un risque sanitaire accru. Les activités professionnelles sont probablement plus à risques pour les garçons; pour les filles, elles impliquent, comme l'accès à l'école, la possibilité de sortir du foyer familial et d'acquérir de nouvelles compétences. Pour les garçons, l'école ne joue pas ce rôle d'ouverture sur le monde extérieur et l'absence de scolarisation n'a pas la même signification en termes de compétences humaines.

\section{Conclusion}

En conclusion, l'approche des Capabilités vise à identifier les groupes et situations plus vulnérables en distinguant le rôle des conditions du contexte de celui des facteurs plus personnels qui diminuent, ou au contraire renforcent, le potentiel individuel et les capacités à transformer ce potentiel en bienêtre. Dans cette optique, la présente analyse a mis en évidence, principalement, quatre dimensions de la vulnérabilité:

- Facteurs de contexte: Les femmes sont plus vulnérables aux facteurs environnementaux, vraisemblablement à travers les tâches domestiques; les hommes aux modes de vie acquis par l'ancrage socio-culturels et au contexte de travail.

- Parcours de vie: La jeunesse est une période de bonne santé, mais l'entrée dans l'âge adulte - pour les femmes, la maternité et, pour les hommes, l'accès au marché du travail - implique des risques particuliers.

- Ressources économiques: On n'observe pas une moins bonne santé parmi les jeunes des familles les plus pauvres de l'échantillon. Au contraire, une certaine aisance et l'accès aux commerces de toutes sortes semble entraîner des comportements masculins néfastes à la santé. Ce différentiel de comportements apparaît cependant estompé par des contacts avec le système de soins.

- Ressources humaines: Des contacts hors du foyer familial, en particulier l'accès à l'école, jouent un rôle central pour que les filles puissent acquérir les compétences pour profiter de l'offre de services, mais pour les deux sexes cette ouverture implique des risques accrus, s'ils ne sont pas compensés par l'acquisition de bonnes connaissances.

Nos résultats confirment donc la thèse de West sur l'absence de gradient socioéconomique simple dans la santé des jeunes. $\mathrm{Ce}$ serait plus les comportements et modes de vie adoptés par les jeunes qui seraient déterminants. Nos résultats montrent surtout les grandes différences de genre à cet égard. Ainsi la thèse de l'influence de la "Culture Jeune» s'appliquerait aux jeunes hommes, alors que pour les jeunes femmes se serait plutôt celle de la vulnérabilité accrue de certains sousgroupes qui serait plus pertinente.

Finalement, notre analyse va bien dans le sens des travaux qui montrent le rôle limité de l'accès au système moderne de soin dans le niveau de santé des populations : ce serait une condition nécessaire mais pas suffisante pour résoudre les problèmes de santé (WHO, 2008). Pour cette population de jeunes, l'utilisation des services de santé n'apparaît pas, en soi, comme une Capabilité, à savoir un facteur d'accroissement réel de la liberté de maintenir une bonne santé. Le recours aux services semble plus conjoncturel - les circonstances s'y prêtent - que relevant d'une plus grande préoccupation de sa santé et il ne gomme pas les inégalités. Pour les filles, un premier contact a un impact bénéfique sur la santé ultérieure uniquement pour celles ayant des ressources humaines et économiques, il semble donc accroître le fossé avec les moins bien loties. 


\section{Références}

Amit-Talai, V. and H. Wulff (eds, 1995), Youth cultures, a crosscultural perspective. Routledge, London, New York.

Berthé, Fatou (2006), Expérience du projet « Chantier Jeunes " en matière d'analyse des ressources et stratégies de la jeunesse face au défi de l'incertitude. Rapport de stage. Master Population et Santé. Institut Supérieur des Sciences Population, Université de Ouagadougou.

Berthé, Fatou, Claudine Sauvain-Dugerdil et Abdoul W. Dieng (2005), Devenir adulte dans un monde en transformation. La vulnérabilité des jeunes maliens liée aux pratiques et aux comportements néfastes à la santé. XXVè Congrès International Population, Tours.

Berthé, Fatou, Claudine Sauvain-Dugerdil, Samba Diop and Bassoutoura Gakou (2008), The impact of the composition of the relational network on the use of modern health facilities by young persons in Mali. Poster Geneva Health Forum.

Blum, Robert W. and Kristin Nelson-Mmari (2004), The health of young people in a global context. Journal Adolescent Health, 35(5), 402-4I8.

Glass Th.A. and M.J. McAtee (2006), Behavioral science at the crossroads in public health : Extending horizons, envisioning the future. Social Science \& Medicine 62, I650-7I.

Griffiths, Paula,William Johnson, Noël Cameron et al (20l3), In urban South Africa, 16 year old adolescents experience greater health inequality than children. Economics and Human Biology II, 502-5I4.

Heggenhougen, H.K, V. Hackethal and P. Vivec (2003), The behavioural and social aspects of malaria and its control, UNDP/World Bank/WHO.

Holstein, B.E., C.Currie, W.Boyce et al (2009), Socio-economic inequality in multiple health complaints among adolescents: international comparative studies in 37 countries.
International Journal Public Health 54, 260270.

Idler, E.L. and Y. Benyamini (1997), Self-Rated Health and Mortality: A Review of TwentySeven Community Studies, J Health \& Social Behavior 38(I), 2I-37.

Keiser, J., J.Utzinger, M. Caldas de Castro et al (2004), Urbanization in sub-Saharan Africa and implication for malaria control. Am J Trop Med Hyg 7I, I I8 - I 27.

Larochelle, C. (2004), Mapping Malaria and Identifying Risk Factors in Mali. Workshop Spatial Perspectives on Analysis for Curriculum Enhancement.

Locoh, Thérèse et Myriam Mouvagha-Sow (2005), Vers de nouveaux modèles familiaux en Afrique de l'Ouest ? XXVè Congrès international de la population, Tours.

Lopez, Alan D, CD. Mathers, M.Ezzati et al (eds, 2006), Global burden of disease and risk factors. OUP and World Bank.

Macedo de Oliveira, Alexandre, Rosalia Mutemba, Juliette Morgan et al. (20II), Prevalence of Malaria among Patients Attending Public Health Facilities in Maputo City, Mozambique. Am. J. Trop. Med. Hyg. 85(6), 1002-1007.

Marmot, M. (2005), Social determinants of health inequalities. The Lancet 365: 10991104.

Marmot, $M$ and RG Wilkinson (eds, 2006), Social determinants of health. OUP.

Njama, D., G.Dorsey, D.Guwatudde et al. (2003), Urban malaria: primary caregivers' knowledge, attitudes, practices and predictors of malaria incidence in a cohort of Ugandan children, Tropical Medicine \& International Health 8(8), 685-692.

Oakes, JM. and JS. Kaufmann (eds, 2006), Methods in social epidemiology. Wiley, San Francisco.

OMS (2008), Combler le fossé en une génération. Instaurer l'équité en santé en agissant sur les déterminants sociaux. Rapport final Commission des déterminants sociaux de la santé. Genève. 
OMS (20I I), Risques pour la santé des jeunes, Aide-mémoire $N^{\circ} 345$. http://www.who.int/mediacentre/factsheets/ fs $345 / \mathrm{fr} /$

Remund, Adrien (20I2), Is young adults' excess mortality a universal phenomenon? Chaire Quetelet. Louvain-La-Neuve.

Remund, Adrien (20I4), La surmortalité des jeunes adultes: réalité individuelle ou artefact des inégalités sociales? Colloque AIDELF, Bari.

Robeyns, Ingrid (2005), The Capability Approach: A Theoretical Survey, J Human Development 6(I), 93-II4.

Robeyns, Ingrid (201I), The Capability Approach, The Stanford Encyclopedia of Philosophy, E.N. Zalta (ed.).

Rossier, Clementine, A. Soura, B. Baya et al (20II), Pauvreté et santé à la périphérie de Ouagadougou. Colloque AIDELF, Ouagadougou.

Safeukui-Noubissi I., S. Ranque, B. Poudiougou et al.(2004), Risk factors for severe malaria in Bamako, Mali, Microbes and Infection 6, 572-8.

Sauvain-Dugerdil, Claudine et Gilbert Ritschard (2005), Temps non structuré et espaces de vie : risques ou opportunités d'une nouvelle liberté ? UIESP, XXVè Congrès International de la Population, Tours (France).

Sauvain-Dugerdil, Claudine et Emily Roulin (20I0): La figure du père, de la mère et des amis dans un contexte en mutation.Composition de l'entourage perçu et réel de jeunes maliens. Colloque AIDELF, Genève.

Sauvain-Dugerdil, Cl., Bassoutoura Gakou, Fatou Berthé, Andoul W. Dieng, Gilbert Ritschard and Mathias Lersch (2008), The start of the "Sexual Transition" in Mali: risks and opportunities. Studies in Family Planning 39(4), 263-280.

Sen, Amartya (1999), Development as Freedom, New York: Knopf Press.

Subramanian, S. V., T. Huijts, M. Avendano (2010), Self-reported health assessments in the 2002 World Health Survey, Bull WHO $88,131-138$.

Thwing, Julie, Jules Mihigo, Alexandra Pataca Fernandes et al (2009), How Much Malaria Occurs in Urban Luanda, Angola? A Health Facility-Based Assessment. Am. J. Trop. Med. Hyg. 80(3), 487-49I.

Uttley, M. and M.H. Crawford (2005),

Biological indication of functioning age: a case study. In Sauvain-Dugerdil $\mathrm{Cl}, \mathrm{H}$. Leridon and N. Mascie-Taylor (eds), Human Clocks. The bio-cultural meanings of Age. Peter Lang, Population, Family and Society V.5, 85- 100 .

West, P. (1997), Health inequalities in the early years: is there equalization in youth ? Social Science and Medicine 44: 833-858.

WHO (2009), Global Health Risks. World Health Organization, Geneva.

' Voir site OMS, santé des adolescents http://www.who.int/topics/adolescent health

iV Voir aussi le programme OMS « Global Burden of Disease " :

http://www.who.int/healthinfo/global burden dis ease/about/en/index.html

iii Projet « Chantier Jeunes » ayant bénéficié de financement du Bureau de la coopération suisse à Bamako pour la phase de terrain et d'un subside du programme conjoint du Fonds National suisse de la recherche scientifique (FNS) et de la Direction de la coopération au développement d'encouragement au partenariat entre Universités suisses et du Sud.

iv Relevé exhaustif des ménages ayant au moins un jeune à Bandiagara Coura, la zone la plus réduite, et dans les deux autres, par Random Route

Sampling, à savoir une sélection de rues à partir d'un point central, puis visite d'une concession sur trois.

${ }^{\vee}$ Voir en particulier Sauvain-Dugerdil et Ritschard (2005), Sauvain-Dugerdil et al, (2008), SauvainDugerdi et Roulin (2010) Berthé (2006), Berthé et al (2005 et 2008) 
vi Voir aussi le chapitre 7 de ce volume qui utilise le module sur la participation sociale de cette même enquête.

vii Réponses à chacun des sept items de la question : A qui vous confiez-vous si vous avez des problèmes de santé, scolaires, au travail, d'argent, avec les amis, avec la famille; si vous êtes tristes, pour partager un secret.

viii Pour plus de détail sur l'entourage des jeunes voir Sauvain-Dugerdil et Roulin 2010 et Dieng, chapitre $7 d u$ présent volume.

ix Il s'agissait de répondre à la question «Quels problèmes ayant affecté votre santé avez-vous eus durant votre vie (ne pas noter les maux périodiques et palu sans gravité) ", complétée par "Avez-vous eu d'autres maladies ou problèmes importants de santé ?» et une question subsidiaire "Avez-vous déjà eu des crises de palu de gravité exceptionnelle (délire et/ou hospitalisation) ? ". Les problèmes étaient enregistrés dans une grille biographique à l'année près et quelques précisions devaient être fournies sur la durée de l'indisposition « Combien de temps avez-vous été empêché de réaliser normalement vos activités ? ", et l'itinéraire thérapeutique: "A qui vous êtes-vous adressé en premier pour vous aider ? " et "Avez-vous reçu des soins ? si oui, où ?».

${ }^{x}$ Le nombre de jours de restriction d'activités étant relevé en 8 catégories, la moyenne a été calculée en imputant à l'individu la valeur centrale de sa catégorie.

${ }^{x i}$ Que pouvez-vous dire sur votre état de santé? très bonne-bonne-moyenne-mauvaise - NR ? Pourquoi?

xii Hôpital, Centre de santé ou médecin privé.

xiii Site OMS, santé des adolescents http://www.who.int/topics/adolescent health

xiv Plus de variables significatives et modèles expliquant une part plus grande de la variabilité (valeurs plus importantes des $\mathrm{R}^{2}$ ).

${ }^{x v}$ Le module sur l'emploi du temps avait effectivement montré que leurs activités les amènent plus souvent à s'éloigner de leur lieu de domicile (25\% d'entre eux avaient fréquenté d'autres quartiers, alors que ce n'était le cas que de $7 \%$ des femmes).

xvi Le samedi précédant l'enquête, les filles ont passé $79 \%$ de leur temps éveillé dans le périmètre de la maison, les garçons $39 \%$. 